\title{
A History of Cosmic Habitability
}

\author{
Amedeo Balbi \\ Physics Department University of Rome Tor Vergata, Italy
}

Correspondence | balbi@romaz.infn.it

Citation | Balbi, Amedeo. 2020. “A History of Cosmic Habitability.” Journal of Big History IV (2). 44-48.

DOI | https://doi.org/10.22339/jbh.v4i2.4220

Abstract Our understanding of the universe is based on the big bang cosmological model, which describes an expanding universe whose development began 13.8 billion years ago from a hot, dense state. This model introduces a strong evolutionary and historical perspective to the account of many observed physical phenomena, including the origin of life and its possible distribution in the universe. I discuss how properly taking the "big picture" and its temporal unfolding into account is relevant for the scope of astrobiology and SETI (the search for extra-terrestrial intelligence).

\section{Introduction}

In the past century, we have learned much about the history of the universe. One of the most important of these is precisely that the universe has a history. This is to say that we know that the observable universe was not always the way it is today. It has been evolving for 13.8 billion years, and it has changed dramatically during this time. It all started in the past from a very simple and undifferentiated state, very close to equilibrium (at least in some sense to be specified later) and then, as time passed, complexity and structure slowly

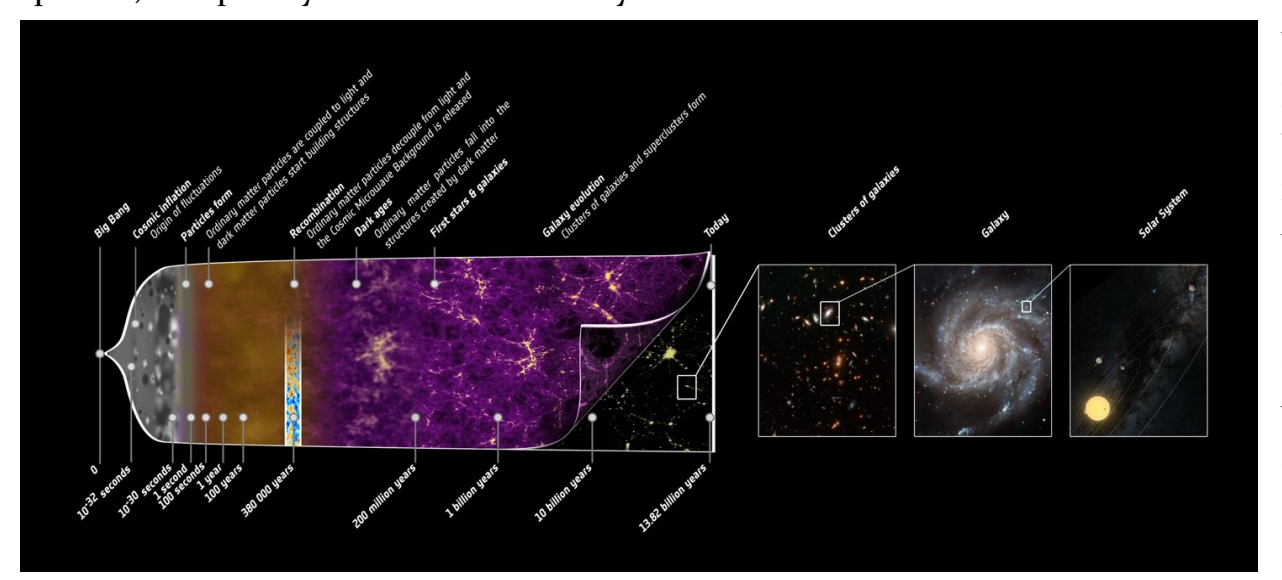

Figure 1: The "big picture" of the history of the universe according to the standard cosmological big bang

model (Image credit: NASA)

emerged (Figure 1). From a hot primordial plasma of free particles, the forces of nature assembled galaxies, stars, planets and molecules. The key questions for astrobiology are: How does life fit into the general scheme of the universe? How common is it? Is life on Earth just a fluke event, without equal in the rest of the universe, or is it part of a generic phenomenon that happened many times elsewhere? The main point of this article is to emphasize how the perspective of big history is essential to attain a good grasp of such issues.
For a physicist or a cosmologist, most of the things that happened in the history of the universe are rather simple to predict. For example, knowing that at the beginning of the universe existed density fluctuations of a given amplitude in the primordial plasma, to predict that after some time stars and galaxies will develop would be rather easy. From a physics standpoint, the fact that the universe is full of stars would not be a surprise even to someone who never had seen a star. When we try to include life in the cosmic picture, however, things are much more complicated. No way is known, at the moment, how to predict from first principles things like the epoch when life first appeared or its frequency and distribution in the universe.

In fact, in a scenario where the universe existed and evolved for 13.8 billion years-that is, in the hot big bang model that constitutes our current best description of cosmic history-even the existence of a single instance of life on one single planet poses a puzzle. To 
understand why, compare the situation with the one envisioned by the now discredited steady-state model (Bondi and Gold 1948), which posits a universe that has been existing forever in the same state as we observe it today: in such a universe, there was enough time (in fact, an infinite amount of time) to produce even the most unlikely combination of molecules, somewhere. So, in a stationary, eternal universe, one should not be too surprised to find that life exists. On the other hand, the mere existence of a single example of life in a universe with a finite age - the kind of universe we, in fact, live in-begs for an explanation. Equally importantly, the evaluation of the likelihood of the appearance of life cannot be easily disentangled from the overall evolution of the cosmos and from its specific features, such as the values of the physical parameters and constants that govern its behavior (Barrow 1998; Rees 1972).

Cosmic History, Complexity and Life In the big bang model, the appearance of life-or, at the very least, the emergence of the conditions for the appearance of life-requires a number of preliminary steps. First, the universe has to create the nuclei of light elements in the primordial plasma; then it has to form stars, and the stars have to cook heavier elements in their nuclei until their abundance is high enough to form rocky planets around main sequence stars. Then, molecular clouds in interstellar space have to form organic molecules that can end up into planetary atmospheres and surfaces, while collisions and impacts with icy bodies have to carry enough water to planets in the habitable zone of their stars-where temperatures and atmospheric pressure are compatible with the presence of lakes and oceans. Only after all these steps have taken place can a chance for replicating molecules appear-and perhaps metabolism, microorganisms, complex life, and intelligence. This is true regardless of the abundance of life in the universe-whether it is widespread or it exists in just a few locations in the whole observable universe. The problem of the origin of life cannot be addressed without having a grasp of this big picture. This is the overall cosmic context where all the necessary steps leading to the appearance of life took place. It is important to note that all such steps can be arranged in a broad evolutionary narrative (Chaisson 2001), and this, in turn, makes the historical perspective crucial to interpret the physical picture.

The very fact that an evolutionary tendency persists may seem counterintuitive at first. The obvious trend from simplicity to complexity that we observe in the universe seems to go against our naïve understanding of thermodynamics: we know that simplicity is more likely than complexity and that disorder comes after order, not vice versa. Actually, however, nothing about this is strange. In fact, the spontaneous appearance of order and selforganization in the universe is possible for two reasons, both of which are di-

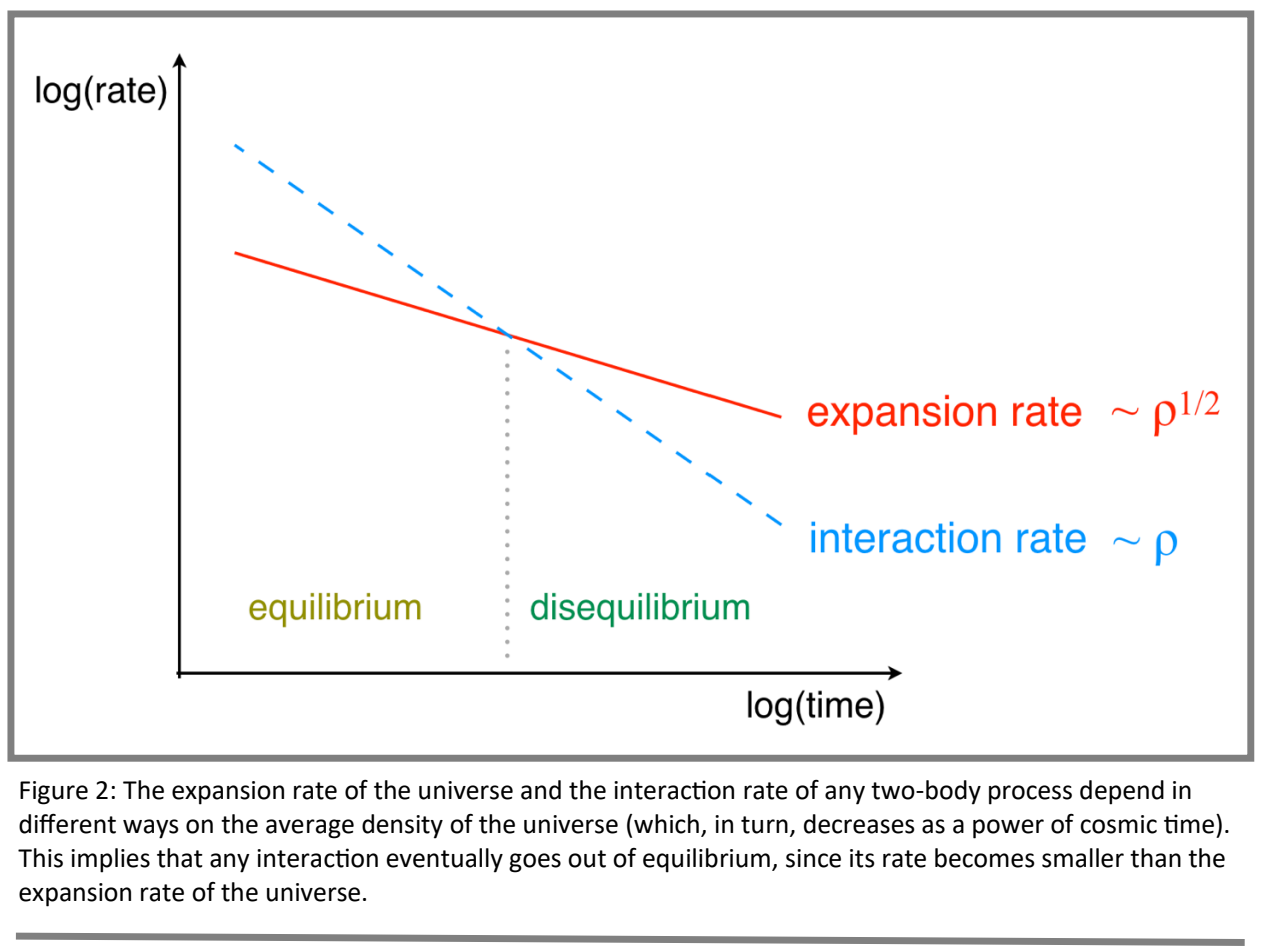

Figure 2: The expansion rate of the universe and the interaction rate of any two-body process depend in different ways on the average density of the universe (which, in turn, decreases as a power of cosmic time). This implies that any interaction eventually goes out of equilibrium, since its rate becomes smaller than the expansion rate of the universe.

rectly related to overall cosmological behavior. First, in an expanding universe, any process tends to go out of equilibrium at some point (Figure 2). This is due to the fact that the average time required for any interaction to take place eventually becomes greater than the rate at which the universe is expanding (i.e., the time it takes for the universe to double its size) because although both of these rates depend upon the overall density of the universe, they depend upon it in different ways (Balbi 2018). When this happens, the interaction cannot be quick enough to maintain equilibrium. Thus, disequilibrium can arise from a very uniform initial state.

The second reason is related to the thermodynamical role of gravity. When we think of entropy, the standard textbook picture is that of a gas in a box (Figure 3). If the gas starts with all the molecules clumped in one corner of the box, we expect that at later times it ends up in a simpler, more uniform and disordered state, where the molecules are evenly spread all over the box. So, we tend to associate high entropy with high uniformity. If one includes gravity 
in the pictures, things go the opposite way: clumpiness and entropy increase in the same direction. In fact, despite the smooth appearance, the initial state of the universe is very far from the highest possible disorder. In thermodynamic parlance, an expanding universe has plenty of ways to increase its entropy while, at the same time, producing transitory complex structures (see, e.g., Carroll 2010).

All of this, of course, is crucial for the possibility of life. Life thrives on the disequilibrium produced by the evolution of the universe. Think, for example, of sunlight striking the surface of Earth. Photons from the Sun have low entropy compared to the ones leaving Earth to space. In the midst of this flux of photons from a low entropy heat source (the Sun) to a higher entropy colder sink (empty space), is Earth and life on it. Life is possible because there is work to be extracted from this thermodynamic engine operating between different temperatures (Lineweaver and Egan 2008). A number of studies have started to adopt an explicitly thermodynamical point of view when considering life in the cosmic context (see, e.g., Frank, Kleidon and Alberti 2017). One way to frame the issue is to see life as an efficient way (or a more efficient way than non-living systems) to use free energy and produce entropy, according to a maximum entropy principle (MEP, see e.g., Kleidon 2010a, 2010b).

We then see a strong historical aspect (in the sense of a deep relation with the flow of time) when we try to understand the place of life in the universe. This is true not just with regard to past cosmic history but also when considering its future evolution. We are not entirely sure of what is going to happen; in fact, we might never be entirely sure (see Krauss and Turner 1999), but if the universe keeps expanding at an acceler-

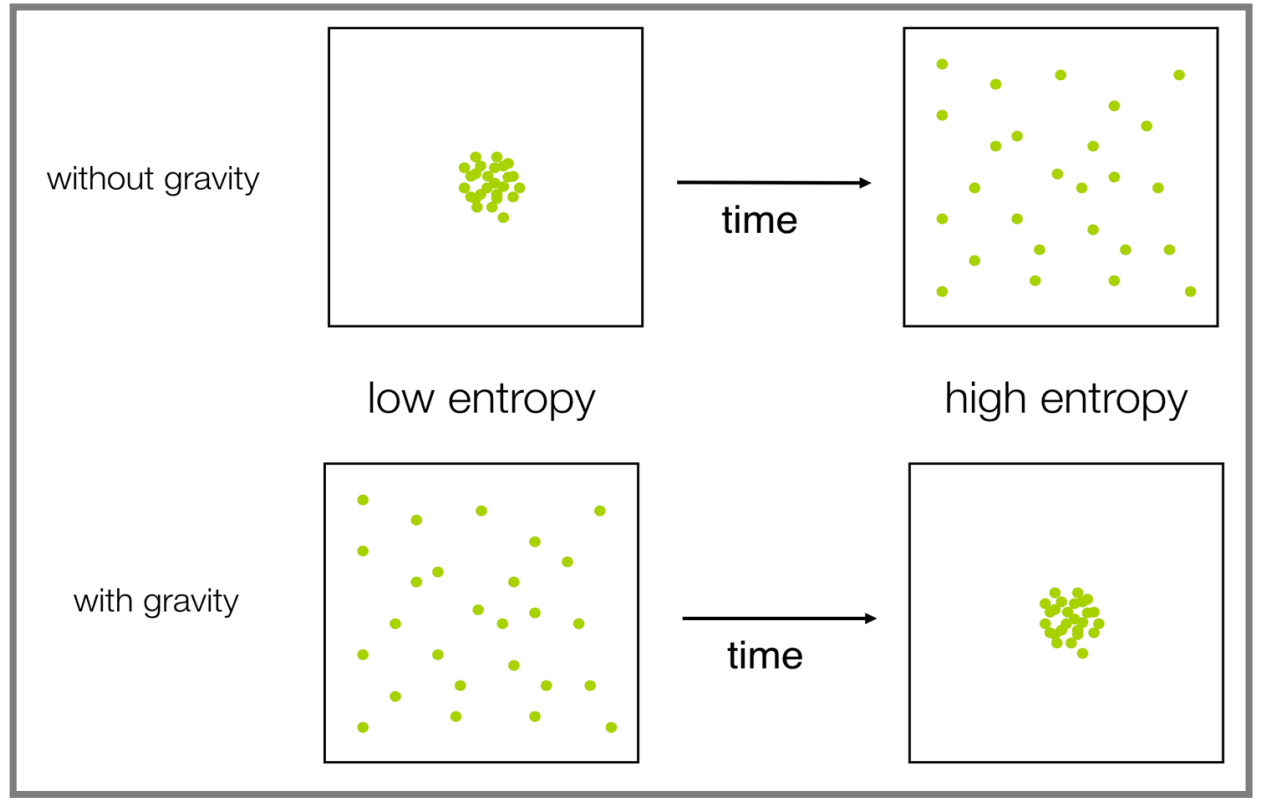

Figure 3: In the presence of gravity, the growth of entropy does not correspond to more uniformity, as in the standard "gas in a box" picture (upper panel): rather, it leads to larger clumpiness (bottom panel). This is crucial to explain the somewhat counterintuitive spontaneous emergence of structure in the universe as time passes.

ated rate (as current observations suggest), eventually it will become cold and structureless, approaching thermodynamic equilibrium (Adams and Laughlin 1997). An eternity will follow where basically nothing complex can happen since the universe will eventually reach "heat death," the highest possible entropy. This puts the present time in an even weirder position. We are living in a fleeting epoch in the overall history of the universe-a tiny temporary interval where there can be stars, planets, molecules and life, enclosed between two equally featureless aeons. One potentially profound consequence of this is that, while Copernicanism is a good guiding principle when applied to space, it is most certainly wrong when applied to time. Our epoch is quite untypical, and we should take this into account when forecasting the frequency and distribution of life in the universe, or judging its likelihood (Cirkovic and Balbi 2019).

\section{When Did the Universe Become Habitable?}

Taking the temporal variable into account, then, is of the utmost im- portance for astrobiology and SETI. One aspect of the role of time is to consider when the universe became suitable for life. This might seem like an obvious question (especially in a big history perspective), but it received surprisingly little attention until not so long ago. In fact, we usually think of habitability only as a question of where the necessary conditions for the origin and maintenance of life can be (see, e.g., Cockell et al 2016). For example, interest abounds in finding planets around the habitable zone of other stars - that is, where the temperature can be compatible with the stable presence of surface liquid water (Kopparapu et al 2013). Some work has also been done to identify suitable locations in our galaxy where habitable planets, and perhaps life, are more likely, i.e., a "galactic habitable zone" (Lineweaver 2001; Lineweaver et al 2004; Gonzales 2005); yet comparatively little effort has been devoted to assessing during what epoch in cosmic history life started being possible (Dayall et al 2016; Loeb et al 2016). It is clear from the previous discussion that the right conditions were not present in our universe from the beginning 
and might not be present in the distant future.

Certain known examples of astrophysical processes with a strong temporal dependence can potentially affect habitability not just on the local scale of planetary systems but even on a galactic scale. For example, we know that during the early history of our galaxy (about eight billion years ago), the supermassive black hole at its center was active-that is, it was accreting matter at a very high rate. As a consequence of this, high levels of ionizing radiation were emitted from the galactic core, and this might have had an impact on the overall habitability of the galaxy. Rocky exoplanets that were close enough to the black hole (within one thousand light years, i.e., in the bulge region of the galaxy) while it was active (a period that might have extended for roughly 100 million years) may have lost a substantial fraction of their atmospheres (Balbi and Tombesi 2017). Moreover, life on such planets might have suffered serious biological damage.

Of course, this is a very large and complex topic, and we are just starting to scratch its surface: it is hard to tell, for example, whether ionizing radiation is only harmful to life or, in some way, also necessary, perhaps to induce mutations and other biogenic effects

(Lingam et al 2019). Clearly, much remains to learn from studying the connection between the history of the universe and its habitability both on small and large scales. Putting together a history of cosmic habitability would be a potentially fecund subfield of big history.

\section{The Temporal Aspects of SETI}

Understanding the history of cosmic habitability would also have a strong impact on the search for intelligent life elsewhere in the universe. Unfortunate- ly, the temporal factor has been almost entirely overlooked in SETI studies (Cirkovic 2004). One area where time enters the game is in the causal properties of any signal that we might be able to detect. In other words, when we search for communicating civilizations that may populate our galaxy, we have to be aware of a strict requirement that any electromagnetic signal (or, more generally, any physical interaction) has to satisfy in order for it to be observed by us today. Just to fix the idea, one can think of a radio message emitted from a location a thousand light-years from the Sun: if such communication ceased before a thousand years ago, we cannot observe it anymore. This may seem at first a trivial fact, but it has profound implications (Balbi 2017; Grimaldi 2017). For example, it implies that any technological civilization of which we might find empirical evidence must be either very long-lived or almost coeval to ours. It also implies that the chances of discovering intelligent life outside Earth depend on how life was distributed in time over the course of cosmic history. If we are latecomers and most other civilizations already have gone extinct, we might be alone. On the other hand, if life started appearing only very recently, and the universe is just waking up right now, we might be one of the infant technological civilizations.

This may shed new light on such old puzzles as the mismatch between the sheer number of potentially habitable locations in the universe and the absence of evidence of intelligent life beyond Earth (a puzzle usually epitomized by Fermi's question: "Where is everybody?"). If the universe was not as life-friendly in the past as it is today, then the questions change and the puzzle takes on new aspects. Evaluating the chances of the success of SETI, or even interpreting negative results, needs to assume knowledge of how the propensity for life of our universe changed over time, a perspective that is inseparable from the historical and evolutionary aspects of the cosmological scenario.

\section{Conclusion}

The fact that we live in an expanding universe with a finite age whose average state changed dramatically over time introduces a historical perspective to many of the physical processes that science tries to explain. I have argued that the problem of the origin of life, on Earth and elsewhere, must fully embrace this historical and evolutionary point of view. Investigating the history of cosmic habitability-i.e., of the propensity of the universe to host life-can illuminate many aspects of contemporary research in both astrobiology and SETI. Eventually, this is yet one more indication that adopting a big history perspective can have profound consequences on understanding our place in the cosmos.

\section{References}

Adams, Fred C., and Gregory Laughlin. 1997. “A Dying Universe: The Long Term Fate and Evolution of Astrophysical Objects." Reviews of Modern Physics 69 (2): 337, doi:10.1103/ RevModPhys.69.337.

Balbi, Amedeo. 2013. "Cosmology and Time." EPJ Web of Conferences 58, doi:10.1051/epjconf/20135802004.

Balbi, Amedeo. 2018. "The Impact of the Temporal Distribution of Communicating Civilizations on Their Detectability." Astrobiology 18 (1): 54.

Balbi, Amedeo, and Francesco Tombesi. 2017. "The Habitability of the Milky Way during the Active Phase of Its Central Supermassive Black Hole." Scientific Reports 7, doi:org/10/1038/ s41598-017-16110-o. 
Barrow, John D. 2008. Cosmology and the Origin of Life. November 30, 1998. Invited presentation. Varenna Conference on the Origin of Intelligent Life in the Universe. ArXiv:astro-ph/9811461.

Bondi, H., and T. Gold. 1948. "The Steady-state Theory of the Expanding Universe.” Monthly Notices of the Royal Astronomical Society 108 (3): 252-270, doi.org/10.1093/ mnras/108.3.252.

Carroll, Sean. 2010. From Eternity to Here: The Quest for the Ultimate Theory of Time. New York: Dutton.

Chaisson, Eric J. 2001, Cosmic Evolution: the Rise of Complexity in Nature. Cambridge, MA: Harvard University Press.

Ćirković, Milan M. 2004. “The Temporal Aspect of the Drake Equation and SETI." Astrobiology 4 (2): 225.

Ćirković, Milan M., and Amedeo Balbi. 2019. "Copernicanism and the Typicality in Time." International Journal of Astrobiology 19 (2): 1-9, doi.org/10.1017/S1473550419000223.

Cockell, C. S., T. Bush, C. Bryce, S. Direito, M. Fox-Powell, J. P. Harrison, H. Lammer, et al. 2016. "Habitability: a Review.” Astrobiology 16 (1): 89-117, doi.org/10.1089/ ast.2015.1295.

Dayal, Pratika, Martin Ward, and Charles Cockell. 2016. "The Habitability of the Universe through 13 Billion Years of Cosmic Time."

ArXiv:16o6.09224.

Frank, Adam, Axel Kleidon, and Marina Alberti. 2017. "Earth as a Hybrid Planet: The Anthropocene in an Evolutionary Astrobiological Context." Anthropocene 19: 13.
Gonzalez, Guillermo. 2005. "Habitable Zones in the Universe." Origin of Life and Evolution of the Biospheres 35

(6): 555-6o6.

Grimaldi, Claudio. 2017. "Signal Coverage Approach to the Detection Probability of Hypothetical Extraterrestrial Emitters in the Milky Way." Scientific Reports 7 (46273).

Kleidon, A. 2010. “A Basic Introduction to the Thermodynamics of the Earth System Far from Equilibrium and Maximum Entropy Production.” Philosophical Transactions of the Royal Society of London. Series B, Biological Sciences. 365 (1545): 1303.

Kleidon, Axel. 2010. "Life, Hierarchy, and the Thermodynamic Machinery of Planet Earth. Physics of Life Reviews 7 (4): 424.

Kopparapu, Ravi Kumar, Ramses Ramirez, James F. Kasting, Vincent Eymet, Tyler D. Robinson, Suvrath Mahadevan, Ryan C. Terrien, et al. 2013. "Habitable Zones around Mainsequence Stars: New Estimates." Astrophysical Journal, 765 (131): 1-16.

Krauss, Lawrence M., and Michael S. Turner. 1999. "Geometry and Destiny." General Relativity and Gravitation 31: 1453.

Lineweaver, C. H. 2001. "An Estimate of the Age Distribution of Terrestrial Planets in the Universe: Quantifying Metallicity as a Selection Effect." Icarus 151: 307-313.

Lineweaver, C. H., Y. Fenner, and B. K. Gibson. 2004. "The Galactic Habitable Zone and the Age Distribution of Complex Life in the Milky Way." Science 303 (5654): 59-62.

Lineweaver, Charles H. 2005. Cosmological and Biological Reproducibil- ity: Limits of the Maximum Entropy Production Principle. In Kleidon, A., and R. Lorenz. Non-equilibrium Thermodynamics and the Production of Entropy: Life, Earth, and Beyond 2: 67.

Lineweaver, Charles H., and Chas A. Egan. 2008. Life, Gravity and the Second Law of Thermodynamics. Physics of Life Reviews 5(4): 225.

Lingam, Manasvi, Idan Ginsburg, and Shmuel Bialy. 2019. "Active Galactic Nuclei: Boon or Bane for Biota?" Astrophysical Journal 877: 62.

Loeb, Abraham, Rafael A. Batista, and David Sloan. 2016. "Relative Likelihood for Life as a Function of Cosmic Time." Journal of Cosmology and Astroparticle Physics 8: 10.

Rees, M. J. 1972. “Cosmological Significance of e2/Gm2 and Related Large Numbers." Comments on Astrophysics and Space Physics 4: 179-185. 